\title{
Building on the success of anti-vascular endothelial growth factor therapy: a vision for the next decade
}

\author{
Anthony P. Adamis ${ }^{1} \cdot$ Christopher J. Brittain ${ }^{1} \cdot$ Atul Dandekar $^{1} \cdot$ J. Jill Hopkins ${ }^{1}$ \\ Received: 16 March 2020 / Revised: 3 April 2020 / Accepted: 14 April 2020 / Published online: 15 June 2020 \\ (c) The Author(s) 2020. This article is published with open access
}

\begin{abstract}
This article aims to identify key opportunities for improvement in the diagnosis and treatment of retinal disease, and describe recent innovations that will potentially facilitate improved outcomes with existing intravitreal vascular endothelial growth factor (VEGF) therapies and lay the groundwork for new treatment approaches. The review begins with a summary of the key discoveries that led to the development of anti-VEGF therapies and briefly reviews their impact on clinical practice. Opportunities for improvements in diagnosis, real-world outcomes with existing therapies, long-acting therapeutics and personalised health care are discussed, as well as the need to identify new targets for therapeutic intervention. Low-cost, remote patient screening and monitoring using artificial intelligence (AI)-based technologies can help improve diagnosis rates and enable remote disease monitoring with minimal patient burden. AI-based tools can be applied to generate patientlevel prognostic data and predict individual treatment needs, reducing the time needed to optimise a patient's treatment regimen. Long-acting therapeutics can help improve visual outcomes by reducing the treatment burden. When paired with AI-generated prognoses, long-acting therapeutics enable the possibility of vision loss prevention. Dual-acting drugs may help improve efficacy and/or durability beyond what is possible with anti-VEGF agents alone. Recent developments and ongoing innovations will help build upon the success of anti-VEGF therapies to further reduce vision loss owing to retinal disease while lowering the overall burden of care.
\end{abstract}

\section{Introduction}

The approval of the first vascular endothelial growth factor (VEGF) inhibitor for neovascular age-related macular degeneration (nAMD) in 2004 heralded in a new era in the treatment of retinal disease. Before the introduction of antiVEGF drugs, the best treatments available for disorders such as nAMD and diabetic macular oedema (DMO) could only slow the rate of vision loss [1, 2]. Anti-VEGF therapy provided the first opportunity to restore functionally meaningful vision in patients with these conditions [3, 4], transforming patients' lives and shifting the goal of treatment from sight preservation alone to sight restoration.

Although anti-VEGF agents have reduced the rates of blindness from nAMD worldwide [5, 6] and will likely have a similar impact on blindness owing to DMO/diabetic

Anthony P. Adamis

adamis.anthony@gene.com

1 Genentech, Inc., South San Francisco, CA, USA retinopathy (DR) [7], these diseases continue to be leading causes of visual impairment [8] and the resultant societal costs remain high [9]. We believe there are several opportunities for improved outcomes in nAMD and DMO/DR. The aims of this paper are to (1) briefly review the history of intravitreal anti-VEGF therapies, and (2) highlight ways we can build upon their success. By leveraging advancements in artificial intelligence (AI), personalised health care, longacting therapeutics and novel drug targets, we believe it is possible to minimise vision loss and achieve better overall management of these diseases over the next decade.

\section{Development of intravitreal VEGF inhibitors}

Beginning in 1948, investigators hypothesised that a diffusible chemical factor emanating from the retina guided the development of the retinal vasculature [10]. This 'Factor X,' as it was termed by George Wise, appeared to be 'intimately associated with the hypoxic retina,' and it was hypothesised to be operative in multiple adult ischaemic retinopathies [11]. The 50-year search for Factor $\mathrm{X}$ ended with the 
discovery of VEGF [12] and the elucidation of its central role in DR [13], DMO [4] and retinal vein occlusion [14]. VEGF was also shown to have a pivotal role in nonischaemic retinal diseases such as nAMD [3, 15] and myopic choroidal neovascularisation [16].

The first anti-VEGF agent for use in the eye, pegaptanib (Macugen, Eyetech, Inc., New York, NY), was approved in 2004. Pegaptanib binds to a subset of VEGF-A isoforms and reduced the average number of letters lost in nAMD by $50 \%$ at 54 weeks [17]. Unlike the standard of care at the time (verteporfin; Visudyne, Bausch and Lomb, Inc., Bridgewater, NJ), pegaptanib was effective in all forms of nAMD [17].

Ranibizumab (Lucentis, Genentech, Inc., South San Francisco, CA) was the first anti-VEGF agent to neutralise all VEGF-A isoforms. It was also the first treatment of any kind to restore functionally meaningful vision in many patients with nAMD and to dramatically reduce the risk of progression to blindness $[3,18]$. In a randomised controlled trial in nAMD [3], the percentage of patients with $20 / 40$ or better vision improved from $4 \%$ at baseline to $39 \%$ after 12 months of monthly ranibizumab $0.5 \mathrm{mg}$, while remaining below $3 \%$ in eyes treated with verteporfin [3]. The rate of legal blindness $(20 / 200$ or worse) in the affected eye decreased from $23 \%$ at baseline to $16 \%$ at month 12 with monthly ranibizumab $0.5 \mathrm{mg}$, while nearly doubling in the verteporfin group (from $32 \%$ to 60\%) [3]. Ranibizumab subsequently became the first anti-VEGF agent approved for the treatment of DMO [4], the leading cause of vision loss in DR, and then for DR itself [16]. In eyes with vision loss due to DMO, the percentage of patients with 20/40 or better vision in the two pivotal studies improved from 14-19\% at baseline to $54-60 \%$ after 12 months of monthly ranibizumab $0.3 \mathrm{mg}$ [4]. Improvements in vision were accompanied by significant improvements in DR severity, and many eyes with proliferative $\mathrm{DR}$ reverted to nonproliferative $\mathrm{DR}$, a less sight-threatening form of the disease [16]. Ranibizumab was also shown to be effective in myopic choroidal neovascularisation, with $>37 \%$ of ranibizumab-treated patients gaining $\geq 15$ letters after 3 months versus $<15 \%$ of photodynamic therapy-treated patients [16].

Aflibercept (Eylea, Regeneron Pharmaceuticals, Inc., Tarrytown, NY) was developed after ranibizumab and inhibits VEGF-A and placental growth factor [19]. Aflibercept produces visual outcomes comparable with ranibizumab in nAMD [19], but the recommended dosing is once every 8 weeks (after three to five monthly loading doses) for nAMD and DMO/DR [19]. Brolucizumab (Beovu, Novartis Pharmaceuticals Corporation, East Hanover, NJ) also was recently approved for nAMD in the United States, demonstrating noninferior visual outcomes compared with aflibercept.

Bevacizumab is an anti-VEGF agent first approved for use in colon cancer in 2004. It is approved for use in a variety of solid tumours, but not for use in the eye [20]. Although marginally less effective than ranibizumab and aflibercept in DMO [21], it is commonly used off label for retinal and choroidal vascular disease.

\section{Impact of anti-VEGF therapies and opportunities for improvement}

Real-world studies in several countries have shown that the adoption of intravitreal anti-VEGF therapy as the standard of care has resulted in a decrease of $\sim 50 \%$ in the number of people becoming legally blind owing to nAMD $[5,6]$. It is also estimated that anti-VEGF therapy could decrease the 2year rate of visual impairment or legal blindness due to DMO by $45 \%$ and $75 \%$, respectively [7]. An epidemiological study in Ireland found that the rate of blindness owing to DR decreased by $~ 50 \%$ from 2011 (30.9 per 100,000 individuals with diabetes) to 2013 (14.9 per 100,000 individuals with diabetes), although the rate of visual impairment owing to DR remained fairly steady (12.3-11.7 per 100,000 individuals with diabetes), highlighting the importance of screening programmes for diabetic eye disease [22].

Despite the impressive impact of anti-VEGF therapies, real-world data indicate that millions of people with nAMD do not achieve the outcomes seen in registrational trials and are still suffering from moderate-to-severe vision impairment or blindness [8].

It is important to understand the root causes of the vision loss that persists and to propose strategies to reduce it. The sections that follow are devoted to this task.

\section{Problem statement: underdiagnosis}

It is estimated that up to half of all patients with DR [23] and up to $25 \%$ of patients with nAMD [24] may be undiagnosed, depriving them of early treatment and increasing their risk of irreversible vision loss [16, 25]. Contributing factors likely include the fact that many people do not visit a doctor regularly, and that the cost of community-based screening programmes using existing methodologies can be high. Although current screening systems in some countries are effective, they are often limited to DMO/DR or glaucoma, and do not cover other retinal diseases such as nAMD.

\section{Potential solution: Al-enabled, low-cost, remote screening}

One potential solution is to use accurate and easy-to-use diagnostic tools to quickly and economically screen large numbers of individuals outside of physicians' offices for any retinal disease. Recent advances in AI and cloud computing may make this possible. 
Deep learning (DL; a type of AI) uses a computer-based neural network that can train itself on a large database to detect an outcome of interest. This approach is being increasingly used in health care to aid diagnosis and gain insights into disease processes [26]. DL is most advanced in the realm of computer vision (evaluation of images). The algorithms can evaluate minute details of millions of high-resolution medical images to accurately diagnose diseases and predict outcomes. Algorithms are more consistent than human experts and may recognise features that are not detectable by human evaluators and/or that had not been suspected of being related to the outcome. This can both increase diagnostic accuracy and provide insights into previously unidentified pathophysiological processes [26]. For example, a recent paper demonstrated that an imaging algorithm could accurately predict age, sex, smoking status and systolic blood pressure from a colour fundus photograph [27], something humans cannot do. In retinal disease, the vast databases of optical coherence tomography (OCT) images and fundus photographs, together with longitudinal data on visual function and treatments, provide a rich resource for training these algorithms.

In April 2018, the US Food and Drug Administration approved the first cloud-based DL algorithm paired with an autonomous retinal fundus camera to automatically identify eyes with DR requiring referral to an ophthalmologist [28]. Using a publicly available set of fundus images and a consensus reference standard set by three US board-certified retinal specialists, the algorithm had a sensitivity of $96.8 \%$, a specificity of $87.0 \%$ and only six out of 874 false negatives [28]. Taking this concept one step further, Arcadu et al. [26] recently built a DL algorithm that predicted OCT measures of DMO from twodimensional colour fundus photographs. Once validated, this algorithm, paired with low-cost digital fundus image capture and cloud computing, could serve as the basis for widespread decentralised screening programmes for DMO, potentially facilitating the timely triage of patients with sight-threatening disease to the care they need to preserve their sight.

\section{Problem statement: suboptimal outcomes in the real world}

Several studies have shown that real-world outcomes with anti-VEGF therapy invariably fall short of those seen in phase 3 anti-VEGF clinical trials; these poorer outcomes are often associated with less frequent treatment $[29,30]$. The need for frequent patient monitoring and intravitreal injections can place a high burden on patients, their caregivers and health care providers [31, 32], and this could be affecting outcomes in clinical practice.

\section{Potential solution: long-acting therapeutics}

One way to relieve the burden of frequent patient monitoring and treatment would be with effective long-acting anti-VEGF therapeutics. Several strategies are actively being investigated, including slow-clearing large molecules, slow-release formulations, continuous delivery drug-device combination technologies and gene therapy [33, 34]. The majority of these technologies are currently in preclinical or early clinical development. However, the Port Delivery System with ranibizumab (PDS) is currently being evaluated in a phase 3 clinical trial [33]. The PDS is a permanent, refillable intravitreal implant for the delivery of ranibizumab for extended durations. In the phase 2 trial, the PDS filled with ranibizumab $100 \mathrm{mg} / \mathrm{mL}$ achieved visual outcomes comparable with monthly ranibizumab injections but with a reduced treatment burden; $~ 80 \%$ of patients did not require a refill for $\geq 6$ months [33]. The median time to first refill was 15 months [33]. Another approach recently approved by the US Food and Drug Administration is the injection of a significantly greater molar concentration of a VEGF inhibitor (brolucizumab).

\section{Problem statement: patient heterogeneity with respect to treatment needs}

The HARBOR [35] and CATT [36] clinical trials demonstrated that the need for anti-VEGF injections varies widely between patients with nAMD. Some patients achieved disease quiescence after a few injections, while others required monthly injections for $\geq 1$ year and still showed signs of disease activity [35]. The majority of patients required between seven to eight injections per year. Treatment needs also can change with time within the same patient [21]. These phenomena underlie the need for frequent office visits. To date, available data sets and traditional statistical methods have been unable to identify factors that could reliably predict treatment need and/or response at the patient level. For both existing and longacting anti-VEGF therapeutics, the ability to predict the optimal treatment frequency at baseline and/or identify disease reactivation remotely will be key to ensuring the best possible treatment outcomes.

\section{Potential solution: remote disease monitoring}

The current standard of care involves frequent bestcorrected visual acuity and OCT monitoring of disease activity in a retinal specialist's office. This is a significant burden for patients, caregivers, health care providers and the health system, especially considering that most patients do not require treatment at every visit. However, if the visit schedule is not frequent enough, detection of disease 
reactivation may be late and vision may be permanently lost owing to delay in treatment. One potential solution is an accurate, economical and user-friendly remote monitoring system; efforts are underway using remote vision testing [37, 38] and remote low-cost OCT. Because remote assessments are conducted more frequently, the signal-tonoise ratio will likely increase, potentially raising the sensitivity and specificity of this approach. One home vision monitoring system recently demonstrated that daily testing can result in early nAMD detection and significantly reduce the risk of vision loss [39].

\section{Potential solution: Al-assisted treatment frequency predictions}

Bogunovic et al. [40] recently demonstrated that patients requiring $\geq 16$ injections over 2 years ('high-needs' patients) could potentially be identified using a DL algorithm. If further refined and validated, this algorithm could allow high-needs patients to be placed on long-acting therapy early, avoiding the burden of multiple frequent evaluations and injections required to determine the patient's treatment needs empirically, and reducing the risk of vision loss in patients who cannot maintain a frequent treatment schedule. Similarly, a DL algorithm could identify low-needs patients who might benefit most from remote monitoring [40], sparing these patients from frequent office visits and greatly reducing the overall burden and cost of care. Using this approach to deliver personalised care will help us move toward optimal vision outcomes with anti-VEGF therapy.

\section{Problem statement: the anti-VEGF-A efficacy ceiling}

Multiple trials have demonstrated that anti-VEGF therapy has reached an efficacy ceiling; specifically, that visual acuity cannot be further improved with greater anti-VEGF-A potency and/or higher doses. For example, in the HARBOR trial, there was no improvement in visual outcomes in patients with nAMD when the ranibizumab dose was increased from $0.5 \mathrm{mg}$ to $2.0 \mathrm{mg}$ [35]. A similar ceiling effect has been seen with other potent anti-VEGF agents in nAMD (e.g., aflibercept, brolucizumab). Peak vision gains are similar across all currently approved agents, with no improvement in outcomes with increasing dose [41, 42]. As a result, several therapies that target VEGF plus a second pathophysiological mechanism are currently in clinical development.

\section{Potential solution: targeting VEGF-A plus a second mechanism}

Since the introduction of anti-VEGF agents for retinal disease $\sim 15$ years ago, no drug targeting a different pathophysiological mechanism has received regulatory approval. The one that is currently at the farthest point in clinical development (phase 3 testing) is the VEGF-angiopoietin (Ang)-2 bispecific antibody, faricimab. Like VEGF, the Ang-2-Tie2 growth factor receptor pathway is operative in normal retinal vascular development [43] and the increased vascular permeability and inflammation seen in DR [44]. In brief, in the normal retina, Ang-1 binding to the Tie2 receptor promotes vascular stability. In the diseased retina, there is a switch to Ang-2 production, promoting destabilisation of the vasculature, subclinical inflammation, vascular leakage and/or neovascularisation [45]. In a phase 2 clinical trial in DMO, simultaneously targeting both VEGF and Ang-2 with faricimab resulted in significantly greater improvements in bestcorrected visual acuity, OCT thickness, DR severity and durability compared with ranibizumab $0.3 \mathrm{mg}$ [46]. Phase 2 clinical trials in nAMD demonstrated that visual outcomes with faricimab every $12-16$ weeks were similar to those with monthly ranibizumab $0.5 \mathrm{mg}$ [47]. Both vision and durability endpoints are being evaluated in the phase 3 programme.

\section{Problem statement: it is not clear who should receive an anti-VEGF monotherapy vs a dual- targeted drug}

If a new dual mechanism of action drug with superior efficacy and/or durability becomes available, it may not be apparent at first diagnosis whether it should be used first or second line. Many patients do well with anti-VEGF monotherapy, achieving good visual outcomes with relatively infrequent treatment. Conversely, some patients still exhibit signs of disease activity and have poor visual function despite frequent injections (i.e., incomplete responders). These patients might have better outcomes if treated with a bispecific antibody as a first-line therapy. Unfortunately, it is not currently possible to identify incomplete responders a priori.

\section{Potential solution: DL algorithms to predict treatment response}

DL proof-of-concept algorithms have been developed that use early functional and anatomic data to predict the treatment response following 12 months of monthly anti-VEGF therapy [48]. If these algorithms can be further improved and validated, it may be possible to identify 'incomplete responders' who would benefit from receiving a bispecific therapy first line. This will allow patients to receive the effective therapy they need without delay and help reduce their risk of irreversible vision loss. Conversely, such an algorithm could help identify patients who will do well with traditional anti-VEGF monotherapy. 

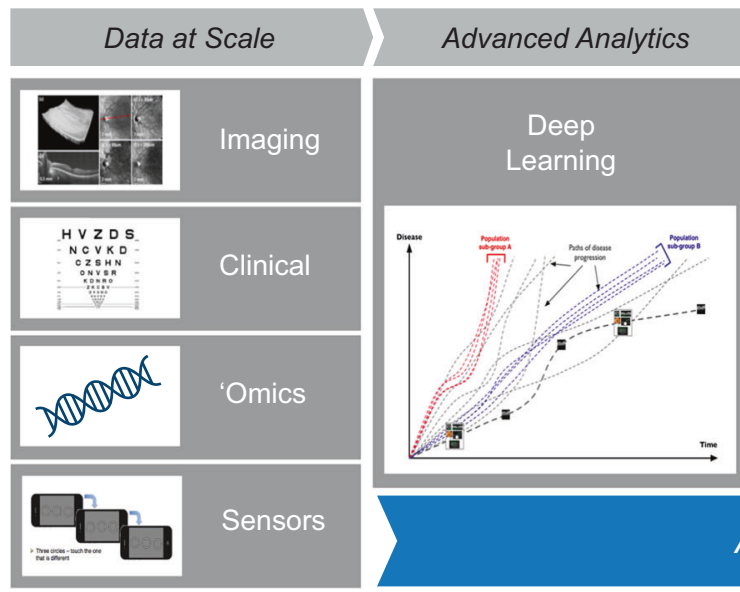

Fig. 1 Use of data and advanced analytics to improve outcomes. Over the next decade, large multidimensional data sets, together with advanced deep learning algorithms, will facilitate the optimal treatment of patients. In addition, prophylaxis of high-risk eyes may

\section{Problem statement: many patients present with irreversible vision loss}

Underdiagnosis, delayed diagnosis and delays in treatment can all lead to irreversible vision loss. Remote screening for active disease can help, but likely will not capture all patients before the onset of irreversible vision loss.

\section{Potential solution: use Al to identify high-risk patients with good vision and treat before vision loss}

A DL algorithm has been created that predicts second eye conversions from intermediate AMD to clinically significant nAMD within 12 months [49]. Once refined and validated, this algorithm could be used together with longacting therapeutics to prevent patients who already have nAMD in one eye from developing bilateral nAMD. Preclinical and clinical studies have shown that continuous anti-VEGF monotherapy can prevent the growth of new [15] and existing choroidal neovascularisation [3, 18]. If eyes with high-risk intermediate AMD can be accurately identified using AI-based tools, the use of safe and minimally invasive long-acting anti-VEGF therapeutics may help prevent the conversion to vision-threatening nAMD.

\section{Conclusions}

In the 15 years since the development of the first intravitreal anti-VEGF therapy, there has been a transformation in the way we treat retinal and choroidal disease. With new tools and platforms such as AI-assisted remote screening and monitoring, predictive algorithms that allow personalised treatment strategies, long-acting therapeutics and new dual-targeted

\section{Personalised Therapy \\ Preservation of Vision}

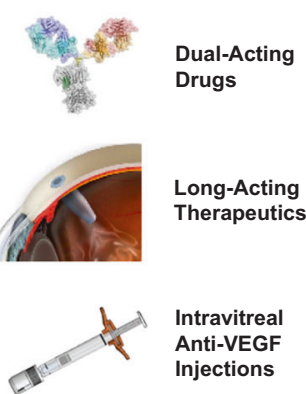

Treat high-risk eyes with good vision.

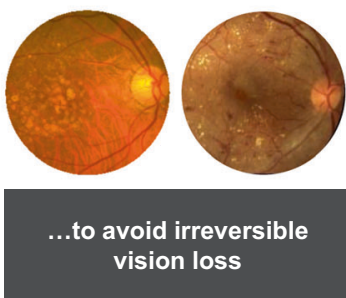

Aspirational goal: NNT = 1 prevent vision loss before any occurs. NNT indicates number of patients that need to receive treatment in order for one patient (on average) to derive benefit; VEGF, vascular endothelial growth factor.

drugs, it may be possible to move closer to a goal of zero blindness due to nAMD and DR in the next decade (Fig. 1). It is hoped that the personalisation of therapy afforded by accurate $\mathrm{AI}$ and remote monitoring will eventually allow the field to approach the coveted 'number needed to treat' of 1 , requiring only a single patient to be treated for every single case of vision loss prevented. This will allow every patient to be assured that they will receive the best treatment plan, at the right time, while also lowering the treatment burden for all involved. This review provides a potential roadmap to be followed in the attempt to achieve these goals.

Acknowledgements Funding was provided by Genentech, Inc. for the study and third-party writing assistance, which was provided by Amy Lindsay, $\mathrm{PhD}$, of Envision Pharma Group.

Funding APA, CJB, AD and JJH are employees of Genentech, Inc.

\section{Compliance with ethical standards}

Conflict of interest The authors are employees of Genentech, Inc.

Publisher's note Springer Nature remains neutral with regard to jurisdictional claims in published maps and institutional affiliations.

Open Access This article is licensed under a Creative Commons Attribution 4.0 International License, which permits use, sharing, adaptation, distribution and reproduction in any medium or format, as long as you give appropriate credit to the original author(s) and the source, provide a link to the Creative Commons license, and indicate if changes were made. The images or other third party material in this article are included in the article's Creative Commons license, unless indicated otherwise in a credit line to the material. If material is not included in the article's Creative Commons license and your intended use is not permitted by statutory regulation or exceeds the permitted use, you will need to obtain permission directly from the copyright holder. To view a copy of this license, visit http://creativecommons. org/licenses/by/4.0/. 


\section{References}

1. Treatment of Age-related Macular Degeneration With Photodynamic Therapy (TAP) Study Group. Photodynamic therapy of subfoveal choroidal neovascularization in age-related macular degeneration with verteporfin: one-year results of 2 randomized clinical trials-TAP report. Arch Ophthalmol. 1999;117:1329-45.

2. Early Treatment Diabetic Retinopathy Study Research Group. Photocoagulation for diabetic macular edema. Early Treatment Diabetic Retinopathy Study report number 1. Arch Ophthalmol. 1985;103:1796-806.

3. Brown DM, Kaiser PK, Michels M, et al; ANCHOR Study Group. Ranibizumab versus verteporfin for neovascular age-related macular degeneration. N Engl J Med. 2006;355:1432-44.

4. Nguyen QD, Brown DM, Marcus DM, et al; RISE and RIDE Research Group. Ranibizumab for diabetic macular edema: results from 2 phase III randomized trials: RISE and RIDE. Ophthalmology. 2012;119:789-801.

5. Sloan FA, Hanrahan BW. The effects of technological advances on outcomes for elderly persons with exudative age-related macular degeneration. JAMA Ophthalmol. 2014;132:456-63.

6. Bloch SB, Larsen M, Munch IC. Incidence of legal blindness from age-related macular degeneration in Denmark: year 2000 to 2010. Am J Ophthalmol. 2012;153:209-13.e2.

7. Varma R, Bressler NM, Doan QV, et al. Visual impairment and blindness avoided with ranibizumab in Hispanic and non-Hispanic whites with diabetic macular edema in the United States. Ophthalmology. 2015;122:982-9.

8. Flaxman SR, Bourne RRA, Resnikoff S, et al; Vision Loss Expert Group of the Global Burden of Disease Study. Global causes of blindness and distance vision impairment 1990-2020: a systematic review and meta-analysis. Lancet Glob Health. 2017;5:e1221-34.

9. Wittenborn J, Rein D. Cost of vision problems: the economic burden of vision loss and eye disorders in the United States. https://www.preventblindness.org/sites/default/files/national/ documents/Economic\%20Burden\%20of\%20Vision\%20Final\% 20Report_130611_0.pdf. Accessed 5 August 2019.

10. Michaelson IC. The mode of development of the vascular system of the retina, with some observations on its significance for certain retinal diseases. Trans Ophthalmol Soc U K. 1948;68:137-80.

11. Wise GN. Retinal neovascularization. Trans Am Ophthalmol Soc. 1956;54:729-826.

12. Leung DW, Cachianes G, Kuang WJ, Goeddel DV, Ferrara N. Vascular endothelial growth factor is a secreted angiogenic mitogen. Science. 1989;246:1306-9.

13. Aiello LP, Avery RL, Arrigg PG, et al. Vascular endothelial growth factor in ocular fluid of patients with diabetic retinopathy and other retinal disorders. N Engl J Med. 1994;331:1480-7.

14. Adamis AP, Shima DT, Tolentino MJ, et al. Inhibition of vascular endothelial growth factor prevents retinal ischemia-associated iris neovascularization in a nonhuman primate. Arch Ophthalmol. 1996;114:66-71.

15. Krzystolik MG, Afshari MA, Adamis AP, et al. Prevention of experimental choroidal neovascularization with intravitreal anti-vascular endothelial growth factor antibody fragment. Arch Ophthalmol. 2002;120:338-46.

16. Lucentis [package insert]. South San Francisco, CA: Genentech, Inc.; 2018.

17. Gragoudas ES, Adamis AP, Cunningham ET Jr., Feinsod M, Guyer DR; VEGF Inhibition Study in Ocular Neovascularization Clinical Trial Group. Pegaptanib for neovascular age-related macular degeneration. N Engl J Med. 2004;351:2805-16.

18. Rosenfeld PJ, Brown DM, Heier JS, et al; MARINA Study Group. Ranibizumab for neovascular age-related macular degeneration. $\mathrm{N}$ Engl J Med. 2006;355:1419-31.
19. Eylea [package insert]. Tarrytown, NY: Regeneron Pharmaceuticals, Inc.; 2019.

20. Avastin [package insert]. South San Francisco, CA: Genentech, Inc.; 2019.

21. Wells JA, Glassman AR, Ayala AR, et al; Diabetic Retinopathy Clinical Research Network. Aflibercept, bevacizumab, or ranibizumab for diabetic macular edema: two-year results from a comparative effectiveness randomized clinical trial. Ophthalmology. 2016;123:1351-9.

22. Tracey ML, McHugh SM, Fitzgerald AP, Buckley CM, Canavan RJ, Kearney PM. Trends in blindness due to diabetic retinopathy among adults aged 18-69 years over a decade in Ireland. Diabetes Res Clin Pract. 2016;121:1-8.

23. Delcourt C, Massin P, Rosilio M. Epidemiology of diabetic retinopathy: expected vs reported prevalence of cases in the French population. Diabetes Metab. 2009;35:431-8.

24. Neely DC, Bray KJ, Huisingh CE, Clark ME, McGwin G Jr., Owsley C. Prevalence of undiagnosed age-related macular degeneration in primary eye care. JAMA Ophthalmol. 2017;135: $570-5$.

25. Singer MA, Awh CC, Sadda S, et al. HORIZON: an open-label extension trial of ranibizumab for choroidal neovascularization secondary to age-related macular degeneration. Ophthalmology. 2012;119:1175-83.

26. Arcadu F, Benmansour F, Maunz A, et al. Deep learning predicts OCT measures of diabetic macular thickening from color fundus photographs. Invest Ophthalmol Vis Sci. 2019;60:852-7.

27. Poplin R, Varadarajan AV, Blumer K, et al. Prediction of cardiovascular risk factors from retinal fundus photographs via deep learning. Nat Biomed Eng. 2018;2:158-64.

28. Abràmoff $\mathrm{MD}$, Lou $\mathrm{Y}$, Erginay $\mathrm{A}$, et al. Improved automated detection of diabetic retinopathy on a publicly available dataset through integration of deep learning. Invest Ophthalmol Vis Sci. 2016;57:5200-6.

29. Ciulla TA, Huang F, Westby K, Williams DF, Zaveri S, Patel SC. Real-world outcomes of anti-vascular endothelial growth factor therapy in neovascular age-related macular degeneration in the United States. Ophthalmol Retina. 2018;2:645-53.

30. Holz FG, Tadayoni R, Beatty S, et al. Multi-country real-life experience of anti-vascular endothelial growth factor therapy for wet age-related macular degeneration. Br J Ophthalmol. 2015;99: 220-6.

31. Varano M, Eter N, Winyard S, Wittrup-Jensen KU, Navarro R, Heraghty J. Current barriers to treatment for wet age-related macular degeneration (wAMD): findings from the wAMD patient and caregiver survey. Clin Ophthalmol. 2015;9:2243-50.

32. Prenner JL, Halperin LS, Rycroft C, Hogue S, Williams Liu Z, Seibert R. Disease burden in the treatment of age-related macular degeneration: findings from a time-and-motion study. Am J Ophthalmol. 2015;160:725-31.e1.

33. Campochiaro PA, Marcus DM, Awh CC, et al; The Port Delivery System with ranibizumab for neovascular age-related macular degeneration: results from the randomized phase 2 Ladder clinical trial. Ophthalmology. 2019;126:1141-54.

34. Al-Khersan H, Hussain RM, Ciulla TA, Dugel PU. Innovative therapies for neovascular age-related macular degeneration. Expert Opin Pharmacother. 2019;20:1879-91.

35. Busbee BG, Ho AC, Brown DM, et al; HARBOR Study Group. Twelve-month efficacy and safety of $0.5 \mathrm{mg}$ or $2.0 \mathrm{mg}$ ranibizumab in patients with subfoveal neovascular age-related macular degeneration. Ophthalmology. 2013;120:1046-56.

36. Maguire MG, Martin DF, Ying GS, et al; Comparison of Agerelated Macular Degeneration Treatments Trials (CATT) Research Group. Five-year outcomes with anti-vascular endothelial growth factor treatment of neovascular age-related macular degeneration: 
the Comparison of Age-Related Macular Degeneration Treatments Trials. Ophthalmology. 2016;123:1751-61.

37. Chew EY, Clemons TE, Bressler SB, et al; Appendix 1 for AREDS2-HOME Study Research Group. Randomized trial of the ForeseeHome monitoring device for early detection of neovascular age-related macular degeneration. The HOme Monitoring of the Eye (HOME) study design - HOME Study report number 1. Contemp Clin Trials. 2014;37:294-300.

38. Shah AR, Gardner TW. Diabetic retinopathy: research to clinical practice. Clin Diabetes Endocrinol. 2017;3:9.

39. Chew EY, Clemons TE, Bressler SB, et al; AREDS2-HOME Study Research Group. Randomized trial of a home monitoring system for early detection of choroidal neovascularization Home Monitoring of the Eye (HOME) study. Ophthalmology. 2014; 121:535-44.

40. Bogunovic H, Waldstein SM, Schlegl T, et al. Prediction of antiVEGF treatment requirements in neovascular AMD using a machine learning approach. Invest Ophthalmol Vis Sci. 2017;58: 3240-8.

41. Dugel PU, Koh A, Ogura Y, et al; HAWK and HARRIER Study Investigators. HAWK and HARRIER: phase 3, multicenter, randomized, double-masked trials of brolucizumab for neovascular agerelated macular degeneration. Ophthalmology. 2020;127:72-84.

42. Heier JS, Brown DM, Chong V, et al; VIEW 1 and VIEW 2 Study Groups. Intravitreal aflibercept (VEGF Trap-Eye) in wet agerelated macular degeneration. Ophthalmology. 2012;119:2537-48.
43. Hackett SF, Wiegand S, Yancopoulos G, Campochiaro PA. Angiopoietin-2 plays an important role in retinal angiogenesis. $\mathrm{J}$ Cell Physiol. 2002;192:182-7.

44. Joussen AM, Poulaki V, Tsujikawa A, et al. Suppression of diabetic retinopathy with angiopoietin-1. Am J Pathol. 2002;160: 1683-93.

45. Regula JT, Lundh von Leithner P, Foxton R, et al. Targeting key angiogenic pathways with a bispecific CrossMAb optimized for neovascular eye diseases [published correction appears in EMBO Mol Med. 2019;11:e10666]. EMBO Mol Med. 2016;8:1265-88.

46. Sahni J, Patel SS, Dugel PU, et al. Simultaneous inhibition of angiopoietin-2 and vascular endothelial growth factor-A with faricimab in diabetic macular edema: BOULEVARD phase 2 randomized trial. Ophthalmology. 2019;126:1155-70.

47. Danzig C, Quezada C, Basu K, et al. Efficacy and safety of faricimab every 16 or 12 weeks for neovascular age-related macular degeneration: STAIRWAY phase 2 results. Invest Ophthalmol. Vis Sci. 2019;60:1212.

48. Haskova Z, Arcadu F, Maunz A, Willis JR, Benmansour F, Prunotto M. Artificial intelligence algorithm that predicts diabetic retinopathy (DR) response to anti VEGF treatment in patients via deep learning (DL). Presented at the 19th Euretina Congress, September 5-8, Paris, France. 2019.

49. Schmidt-Erfurth U, Waldstein SM, Klimscha S, et al. Prediction of individual disease conversion in early AMD using artificial intelligence. Invest Ophthalmol Vis Sci. 2018;59:3199-208. 